Artículo

\title{
Modelos de regresión segmentada para estimar el tamaño óptimo de la parcela experimental con caña de azúcar
}

\author{
Luz Elena Barrantes Aguilar \\ Adrián González Estrada ${ }^{\S}$ \\ Miguel Ángel Martínez Damián \\ Ramón Valdivia Alcalá \\ Autor para correspondencia: adrglez@prodigy.net.mx.
}

\section{Resumen}

El objetivo de esta investigación fue estimar el tamaño óptimo de parcela experimental con caña de azúcar en la Región Brunca de Costa Rica. Se utilizaron modelos de regresión segmentada con los datos obtenidos de un ensayo de uniformidad (40 surcos de 84 metros de largo con una separación entre cada uno de 1.5 metros, para un total de $5040 \mathrm{~m}^{2}$ de área experimental), el trabajo de campo se realizó en la finca El Porvenir en Pérez Zeledón Costa Rica, entre el año 2018 y 2019. Los coeficientes de los modelos de regresión lineal con constante (LRP) y de regresión cuadrática con constante (QRP) fueron estadísticamente significativos. Se concluyó que el tamaño óptimo de parcela que minimiza el error experimental para los ensayos que se establezcan en la región, deberían estar en el rango de 72 a $93 \mathrm{~m}^{2}$.

Palabras clave: eficiencia de la investigación agrícola, heterogeneidad del suelo, minimización del error experimental.

Recibido: mayo de 2020

Aceptado: julio de 2020 


\section{Introducción}

En Costa Rica existen siete zonas productoras de caña y trece ingenios azucareros. De acuerdo con el Plan Anual Operativo (PAO) de DIECA (Dirección de Investigación y Extensión de la Caña de Azúcar) en el año 2018, aproximadamente $22 \%$ de los ensayos vigentes se encontraban en la Región Brunca. Entre los temas de investigación más importantes que se estudian en esa región están: la evaluación de variedades, la interacción de nutrientes y la dosificación nutricional, los madurantes, los herbicidas y la densidad de siembra.

Las variables objetivo son frecuentemente el rendimiento de campo y el rendimiento agroindustrial. Para este tipo de investigaciones en la región se han utilizado principalmente tres tamaños de parcela: 5 surcos de $10 \mathrm{~m}$ con una separación de $1.5 \mathrm{~m}\left(75 \mathrm{~m}^{2}\right)$, también se trabaja con 6 surcos de 9 metros con una separación de $1.5 \mathrm{~m}\left(81 \mathrm{~m}^{2}\right)$ o bien, 5 surcos de $8 \mathrm{~m}$ con una separación de $1.5 \mathrm{~m}$ $\left(60 \mathrm{~m}^{2}\right)$, pero nunca se ha validado la eficiencia de esos tamaños de parcela.

Tampoco se ha investigado el tamaño de la parcela experimental con el que se minimiza el error experimental. Por esta razón, el objetivo de este trabajo es encontrar el tamaño óptimo de la parcela experimental con caña de azúcar en la región Brunca de Costa Rica. Sripathi et al. (2017) agrupan, dentro de lo que llaman factores de diseño experimental, al tamaño de la parcela, tamaño del bloque y número de repeticiones y afirman que en los ensayos de rendimiento el error experimental es sensible a esos factores, debido a que la investigación agronómica depende de los datos registrados en los ensayos de campo.

En la definición del tamaño de la parcela experimental influyen muchos factores y los más importantes son: el error experimental y los costos asociados. El tamaño de la parcela debe permitir captar satisfactoriamente toda la heterogeneidad del suelo, considerada la principal fuente de variabilidad de la parcela experimental. Cocco et al. (2009) compararon los resultados que se obtuvieron con distintos tamaños y formas de parcela experimental en el cultivo de fresa sembrada en suelo y bajo el sistema de hidroponía. El cultivo en el suelo presentó mayor variabilidad experimental que el cultivo hidropónico. El desarrollo de los métodos para la estimación del tamaño óptimo de parcela tiene una base importante con el trabajo pionero de Smith (1938), quien encontró una relación asintótica negativa entre la varianza y el tamaño de la parcela. Son muchas las propuestas hechas desde entonces.

Investigaciones actuales fueron en gran parte desarrollados en Brasil y aplicados en diversos cultivos, por ejemplo: crotalaria (Crotalaria juncea L.) (Facco et al., 2017), berenjena (Solanum melongena) (Krysczun et al., 2018), tomate cherry (Solanum lycopersicum L.) (Giacomini y Lúcio, 2018), tomate (Solanum lycopersicum), habichuelas (Phaseolus vulgaris) y calabacín (Cucurbita pepo) (Schwertner et al., 2015), girasol (Helianthus annus L.) (Santos et al., 2015), café (Coffea) (Mendes et al., 2016), taro (Colocasia esculenta) (Silva, 2014) y boniato (Ipomoea batatas) (Rodríguez et al., 2018).

Algunos trabajos recientes también se han realizado en la India, con la mostaza de la India (Brassica juncea L.) (Khan y Tanwar, 2017). En Estados Unidos de América, con sandía (Citrullus lanatus) (Boyhan, 2013). En Costa Rica este tipo de investigación se han realizado para el cultivo de café, a inicios de los sesenta, (Paez, 1962) y para frijol en los años setenta (Mamani, 1971). 
Los ensayos más recientes se realizaron en la zona de Bagaces, Guanacaste, para los cultivos de arroz (Oryza sativa) (Vargas y Navarro, 2014) y maíz (Zea mays) (Vargas y Navarro, 2017). Para el cultivo de la caña de azúcar en otros países ya se ha trabajado en el cálculo del tamaño óptimo de la parcela experimental. Por ejemplo: Igue et al. (1991) en Brasil; Bose y Khanna (1939) en la India y Palencia (1965) y Álvarez (1982) en Guatemala.

\section{Materiales y métodos}

\section{Modelos de regresión segmentada}

Dentro de las propuestas metodológicas para la estimación del tamaño óptimo de la parcela experimental, se encuentran los modelos de regresión segmentada. Paranaiba et al. (2009) proponen ajustar un modelo de regresión lineal con constante (LRP) para la relación entre el coeficiente de variación (CV) del rendimiento y el tamaño de parcela (medido en unidades experimentales básicas (UEB)), tal como se aprecia en la Figura 1, donde para los valores $\mathrm{x}_{\mathrm{i}} \leq \mathrm{x}_{0}$ esta relación está descrita por un modelo lineal decreciente hasta cierto punto, después se vuelve constante. El valor CVP que corresponde al punto de inflexión, se obtiene de un proceso iterativo.

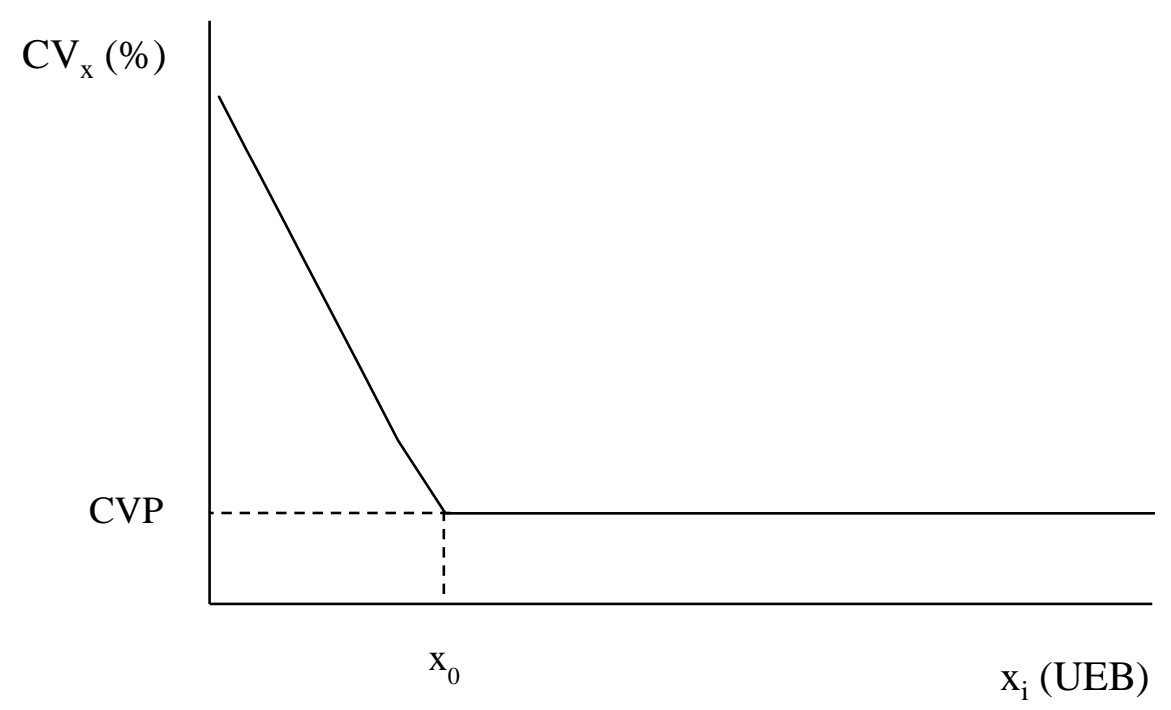

Figura 1. Relación entre el tamaño de la parcela y el coeficiente de variación para el método de segmentación lineal con constante.

Este modelo de regresión lineal segmentada se puede expresar como: $C V_{x}=\left\{\begin{array}{cl}\beta_{0}+\beta_{1} x_{i}+\varepsilon_{i}, & \text { si: } x \leq x_{0} \\ C V P+\varepsilon_{i}, & \text { si: } x>x_{0}\end{array}\right.$ 1) donde: $\mathrm{CV}_{\mathrm{x}}$ es el coeficiente de variación entre los totales para parcelas con $x_{i}$ unidades básicas; CVP es el coeficiente de variación en el punto donde se unen los dos segmentos; $x$ es el tamaño de parcela medido en unidades básicas; y $\varepsilon_{\mathrm{i}}$ es el error asociado a $\mathrm{CV}_{\mathrm{x}}$, supuestamente normal e independientemente distribuido, con media cero y varianza constante.

Dado que los dos segmentos de (1) son iguales en el punto $x_{0}$, entonces: $\beta_{0}+\beta_{1} x_{0}=C V P 2$ ). Por lo que el tamaño óptimo de la parcela experimental está dado por: $\mathrm{x}_{0}=\frac{\mathrm{CVP}-\beta_{0}}{\beta_{1}} 3$ ). 
Otra alternativa dentro de las posibilidades de los modelos segmentados es el método de regresión cuadrática con constante (QRP), descrito por Ferreira (2007) y aplicado por Mendes et al. (2016) para la estimación del tamaño de la parcela experimental. Con respecto al modelo anterior, este método asume una forma polinomial de segundo grado, en lugar de una forma lineal en el primer segmento (Figura 2).

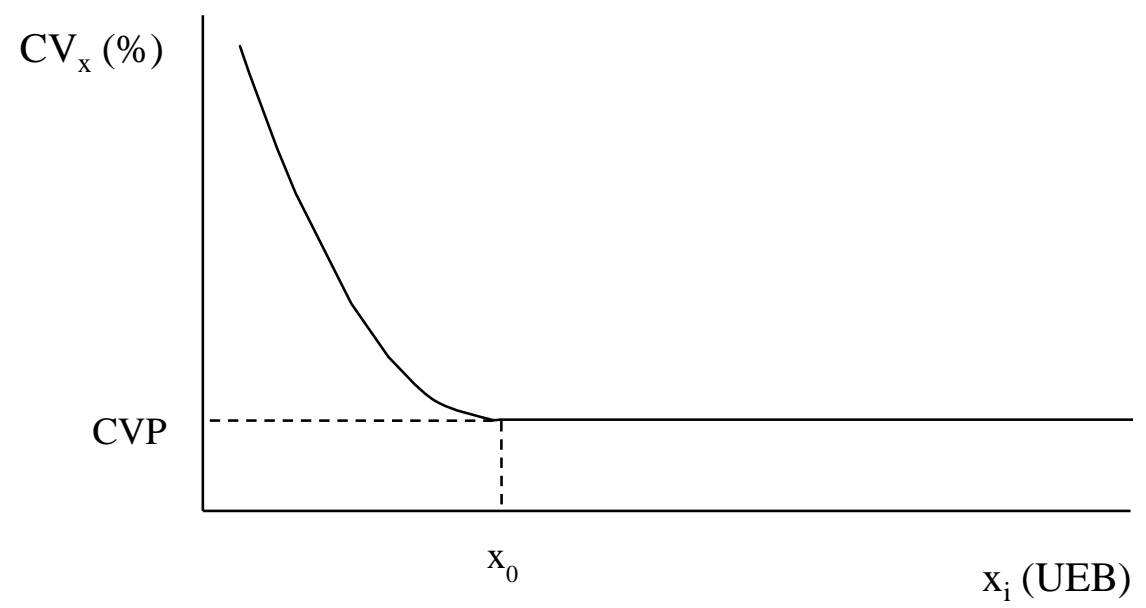

Figura 2. Relación entre el tamaño de la parcela y el coeficiente de variación para el método de segmentación cuadrática con constante.

Para valores $\mathrm{x}_{\mathrm{i}} \leq \mathrm{x}_{0}$ el modelo es cuadrático y para valores $\mathrm{x}_{\mathrm{i}}>\mathrm{x}_{0}$ es constante. De igual manera, la intercepción entre los segmentos determina el tamaño óptimo: $C V_{x}=\left\{\begin{array}{cl}\beta_{0}+\beta_{1} x_{i}+\beta_{2} x_{i}^{2}+\varepsilon_{i}, & \text { si: } x \leq x_{0} \\ C V P+\varepsilon_{i}, & \text { si: } x>x_{0}\end{array}\right.$ 4). El punto $x_{0}$ representa el punto de unión de los dos segmentos y se debe estimar juntamente con los demás parámetros del modelo. Dado que la curva debe ser continua y suavizada, las primeras derivadas con respecto a $x$ en ambos segmentos deben ser la misma para el punto $x_{0}$. De acuerdo con Ferreira (2007), esta condición implica que: $\frac{\partial C V_{x}}{\partial x}=\beta_{1}+2 \beta_{2} x_{i}$ 5). Una vez igualada a cero, la ecuación (5) se resuelve para $x$ y después de sustituir $x$ por $x_{0}$, se obtiene: $x_{0}=\frac{-\beta_{1}}{2 \beta_{2}} \quad 6$ ); El valor de la constante (CVP) se puede obtener al sustituir este valor en la ecuación (4): $\left.\mathrm{CV}_{\mathrm{x}}=\mathrm{CVP}=\beta_{0}+\beta_{1} \mathrm{x}_{0}+\beta_{2} \mathrm{x}_{0}^{2}=\beta_{0}+\beta_{1}\left(\frac{-\beta_{1}}{2 \beta_{2}}\right)+\beta_{2}\left(\frac{-\beta_{1}}{2 \beta_{2}}\right)^{2}=\beta_{0}-\frac{\beta_{1}^{2}}{4 \beta_{2}} 7\right)$

\section{Fuente de información y ensayo de uniformidad}

Se utilizaron los datos de un ensayo de uniformidad de caña de azúcar realizado entre 2018 y 2019 en la finca El Porvenir en Pérez Zeledón, Costa Rica, localizada a una altura de $591 \mathrm{msnm}$. En el año 2017 se registró una precipitación anual de $3673.8 \mathrm{~mm}$ y la temperatura promedio fue de 23.3 ${ }^{\circ} \mathrm{C}$, con una máxima de $34.5^{\circ} \mathrm{C}$ y una mínima de $15.4{ }^{\circ} \mathrm{C}$.

Se plantaron de forma manual 40 surcos de $84 \mathrm{~m}$ de largo con una separación entre surco de $1.5 \mathrm{~m}$ de la variedad RB 99-381 a una densidad de tres chorros. En total fueron $4800 \mathrm{~m}^{2}$ de parcela útil, la cual se dividió en 1600 unidades experimentales básicas (UEB), cada una de $2 \mathrm{~m}$ de largo por 1.5 metros de ancho $\left(3 \mathrm{~m}^{2}\right)$. 
Las prácticas del cultivo que se realizaron fueron las mismas que se aplican a las plantaciones comerciales de la región. Un mes antes de la siembra se preparó el terreno. La siembra se realizó manualmente colocando tres cañas con un traslape de $15 \mathrm{~cm}$ y se utilizan $13 \mathrm{t} \mathrm{ha}^{-1}$ de semilla. Para el control de malezas se empleó una mezcla química de Pendimetalina 50 EC $\left(3 \mathrm{~L} \mathrm{ha}^{-1}\right)$ con Terbutilazina $50 \mathrm{SC}\left(2 \mathrm{~L} \mathrm{ha}^{-1}\right)$ en preemergencia de la maleza (suelo desnudo). Y en post emergencia temprana, una mezcla química de Hexazinona 75 WG $\left(0.5 \mathrm{~kg} \mathrm{ha}^{-1}\right)$ con Diurón 80 WG $\left(2 \mathrm{~kg} \mathrm{ha}^{-1}\right)$.

Por ser caña de primer corte se aplicó $140 \mathrm{~kg}$ de nitrógeno, $140 \mathrm{~kg}$ de fósforo, $167 \mathrm{~kg}$ de potasio, 35 $\mathrm{kg}$ de magnesio y $40 \mathrm{~kg}$ de azufre por hectárea, distribuidos en tres fertilizaciones. Para la cosecha se consideran características de topografía, tamaño y distribución de las fincas de caña en la región, según lo cual se realiza manual o semi mecanizada. La cosecha se realizó de forma manual los días 6 y 7 de marzo de 2019 a los 10 meses de edad de la plantación. La variable que se midió fue el rendimiento de campo, tomando el peso de cada UEB medido en kilogramos.

Las unidades experimentales básicas se agruparon en unidades secundarias, de distintas formas y tamaños tomando como requisito que esas agrupaciones de parcelas adyacentes emplearan siempre la totalidad del área experimental, tal como lo describen Paranaiba et al. (2009), a cada una de estas unidades secundarias se les calculó la media de la producción, la varianza y el coeficiente de variación. Para la estimación del tamaño óptimo de parcela se emplearon los métodos de regresión lineal con constante (LRP) y regresión cuadrática con constante (QRP). En la estimación de los parámetros y el análisis de la información se empleó el paquete estadístico SAS versión 9.3 y Python versión 3 (2008).

\section{Resultados y discusión}

Al momento de la cosecha y pesada del ensayo, de las 1600 unidades experimentales básicas se obtuvo una producción promedio de $19.9 \mathrm{~kg}$ por unidad experimental básica, con una desviación estándar de $3.8 \mathrm{~kg}$. Los valores oscilaron entre $9.5 \mathrm{~kg}$ y $38 \mathrm{~kg}$. Tal como se observa en la Figura 3 , $41 \%$ de las unidades experimentales básicas pesaron entre 18 y $22 \mathrm{~kg}$.

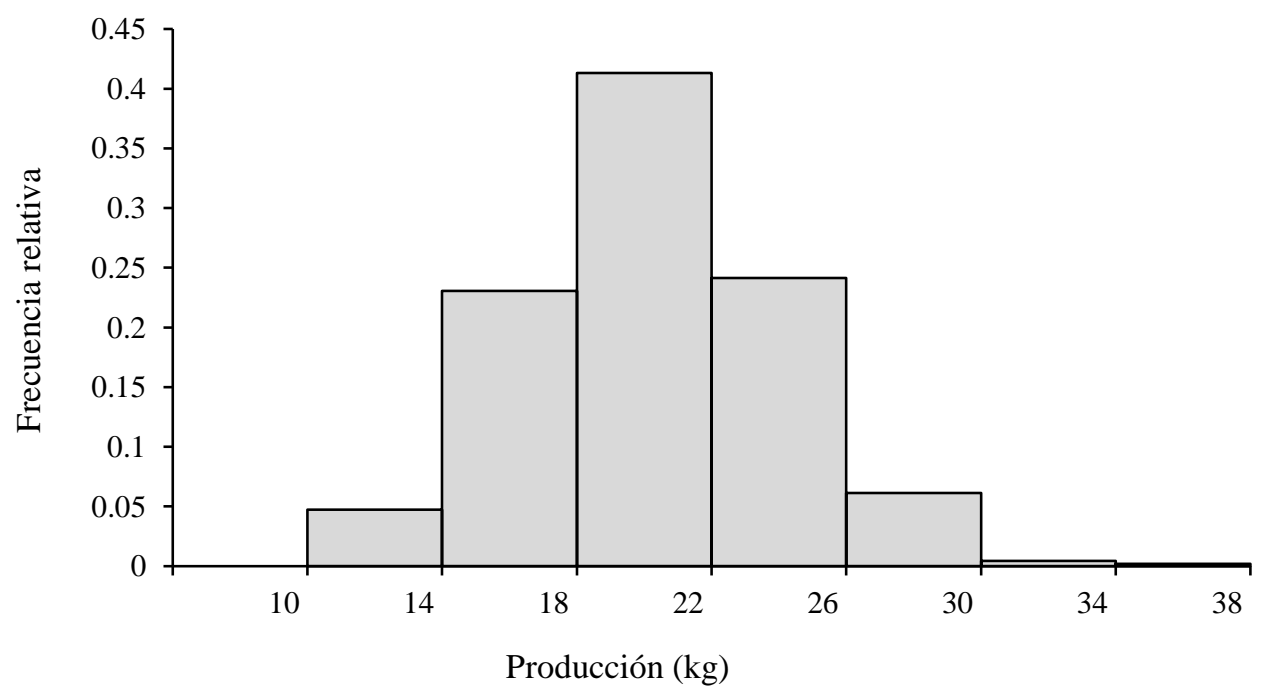

Figura 3. Histograma de la producción obtenida del ensayo de uniformidad. 
En total se agruparon los datos del ensayo en 20 tamaños que corresponden a 63 formas distintas (Cuadro 1).

Cuadro 1. Cantidades y tamaños de parcelas secundarias en las que fue posible agrupar los datos del ensayo.

\begin{tabular}{|c|c|c|c|}
\hline \multirow{2}{*}{$\begin{array}{c}\text { Cantidad de } \\
\text { parcelas } \\
\text { secundarias }\end{array}$} & \multicolumn{2}{|c|}{ Tamaño } & \multirow{2}{*}{ Formas } \\
\hline & UEB & $\mathrm{m}^{2}$ & \\
\hline 1600 & 1 & 3 & $(1 \times 1)$ \\
\hline 800 & 2 & 6 & $(1 \times 2),(2 \times 1)$ \\
\hline 400 & 4 & 12 & $(1 \times 4),(4 \times 1),(2 \times 2)$ \\
\hline 320 & 5 & 15 & $(1 \times 5),(5 \times 1)$ \\
\hline 200 & 8 & 24 & $(1 \times 8),(8 \times 1),(2 \times 4),(4 \times 2)$ \\
\hline 160 & 10 & 30 & $(1 \times 10),(10 \times 1),(2 \times 5),(5 \times 2)$ \\
\hline 100 & 16 & 48 & $(4 \times 4),(2 \times 8),(8 \times 2)$ \\
\hline 80 & 20 & 60 & $(1 \times 20),(20 \times 1),(2 \times 10),(10 \times 2),(4 \times 5),(5 \times 4)$ \\
\hline 64 & 25 & 75 & $(5 \times 5)$ \\
\hline 50 & 32 & 96 & $(4 \times 8),(8 \times 4)$ \\
\hline 40 & 40 & 120 & $(1 \times 40),(40 \times 1),(2 \times 20),(20 \times 2),(4 \times 10),(10 \times 4),(5 \times 8),(8 \times 5)$ \\
\hline 32 & 50 & 150 & $(5 \times 10),(10 \times 5)$ \\
\hline 25 & 64 & 192 & $(8 \times 8)$ \\
\hline 20 & 80 & 240 & $(2 \times 40),(40 \times 2),(4 \times 20),(20 \times 4),(8 \times 10),(10 \times 8)$ \\
\hline 16 & 100 & 300 & $(5 \times 20),(20 \times 5),(10 \times 10)$ \\
\hline 10 & 160 & 480 & $(4 \times 40),(40 \times 4),(8 \times 20),(20 \times 8)$ \\
\hline 8 & 200 & 600 & $(5 \times 40),(40 \times 5),(10 \times 20),(20 \times 10)$ \\
\hline 5 & 320 & 960 & $(8 \times 40),(40 \times 8)$ \\
\hline 4 & 400 & 1200 & $(10 \times 40),(40 \times 10),(20 \times 20)$ \\
\hline 2 & 800 & 2400 & $(20 \times 40),(40 \times 20)$ \\
\hline
\end{tabular}

La relación entre el coeficiente de variación de cada de estas formas y el tamaño de la parcela experimental se aprecia en la Figura 4 (para una mejor apreciación se cortó el eje de las abscisas en 400), como se esperaba el coeficiente de variación decrece rápidamente en el segmento de parcelas pequeñas y después, conforme el tamaño aumenta, el coeficiente de variación tiende a disminuir menos que proporcionalmente. 


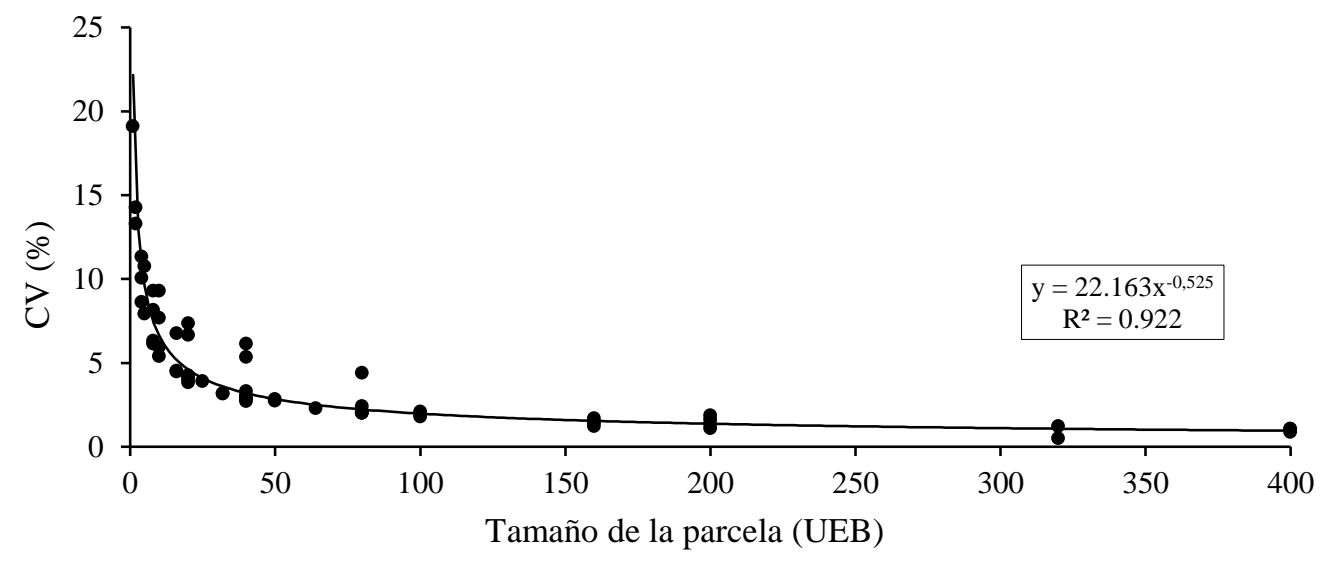

Figura 4. Relación entre el coeficiente de variación (CV) de la producción con el tamaño de la parcela medido en UEB.

Al aplicar el modelo de regresión lineal segmentado (LRP), se obtuvieron los parámetros del modelo: $\mathrm{CV}_{\mathrm{x}}=\left\{\begin{array}{ccc}12.61-0.43 \mathrm{x}_{\mathrm{i}} & \text { si: } \mathrm{x} \leq 24.05 \\ 2.17 & \text { si: } \mathrm{x}>24.05 & 8 \text { ). El coeficiente de determinación fue de } 61.62 \% \mathrm{y}\end{array}\right.$ el estadístico $\mathrm{F}$ del análisis de varianza para la prueba de significancia de los parámetros estimados también fue significativo $(p<0.01)$ (Cuadro 2).

Cuadro 2. Resultados del modelo de regresión lineal con constante (LRP) y del modelo de regresión cuadrática con constante $(\mathrm{QRP})$ para el ensayo de caña de azúcar.

\begin{tabular}{|c|c|c|c|c|}
\hline Parámetros & $\operatorname{LRP}^{(1)}$ & & $\mathrm{QRP}^{(1)}$ & \\
\hline \multirow[t]{2}{*}{$\widehat{\beta}_{0}$} & 12.6093 & $* *$ & 14.1172 & $* *$ \\
\hline & $(0.6728)$ & & $(0.8055)$ & \\
\hline \multirow[t]{2}{*}{$\widehat{\beta}_{1}$} & -0.4338 & $* *$ & -0.7654 & ** \\
\hline & $(0.0537)$ & & $(0.1149)$ & \\
\hline \multirow[t]{2}{*}{$\hat{\beta}_{2}$} & & & 0.0123 & $* *$ \\
\hline & & & $(0.0031)$ & \\
\hline $\mathrm{n}$ & 63 & & 63 & \\
\hline $\mathrm{F}$ & 114.3413 & & 136.2508 & \\
\hline $\mathrm{R}^{2}$ ajustado & 61.62 & & 76.96 & \\
\hline $\mathrm{W}^{(2)}$ & 0.9276 & & 0.9309 & \\
\hline $\mathrm{P}$ & 2.17 & & 2.2256 & \\
\hline $\mathrm{X}_{0}$ & 24.0531 & & 31.0742 & \\
\hline
\end{tabular}

${ }^{(1)}=$ error estándar entre paréntesis; ${ }^{* *}=$ significativo al $1 \% ;{ }^{*}=$ significativo al $5 \% ;{ }^{(2)}=$ estadísticas de prueba ShapiroWilk.

El tamaño óptimo estimado es aproximadamente de 25.05 UEB. Considerado que cada UEB es de $3 \mathrm{~m}^{2}$, el tamaño óptimo equivale a $72.16 \mathrm{~m}^{2}$ (Figura 5). 


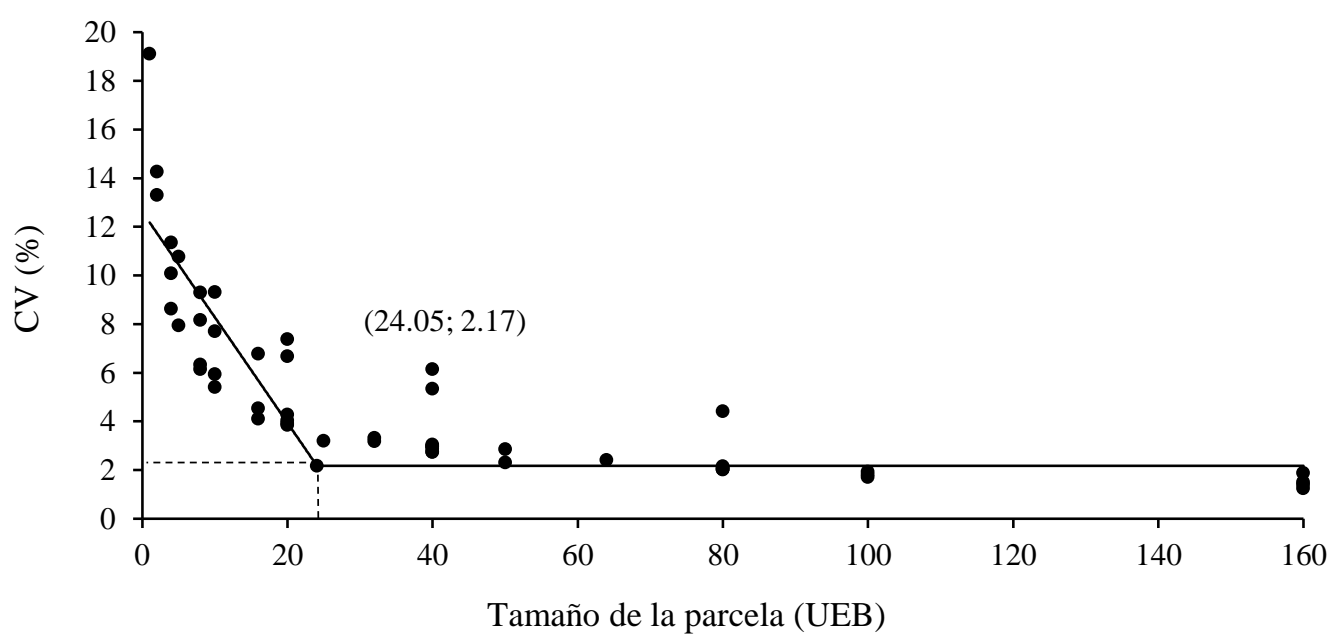

Figura 5. Ajuste mediante el modelo de regresión lineal con constante (RLP).

Con base a los datos para ajustar el modelo de regresión cuadrática con constante, se obtuvieron los estimadores: $\mathrm{CV}_{\mathrm{x}}=\left\{\begin{array}{cc}14.12-0.77 \mathrm{x}_{\mathrm{i}}+0.01 \mathrm{x}_{\mathrm{i}}^{2} & \text { si: } \mathrm{x} \leq 31.07 \\ 2.3 & \text { si: } \mathrm{x}>31.07\end{array}\right.$ 9). En este caso, el tamaño óptimo fue de $31.07 \mathrm{UEB}$, equivalente a parcelas de $93.22 \mathrm{~m}^{2}$ (Figura 6).

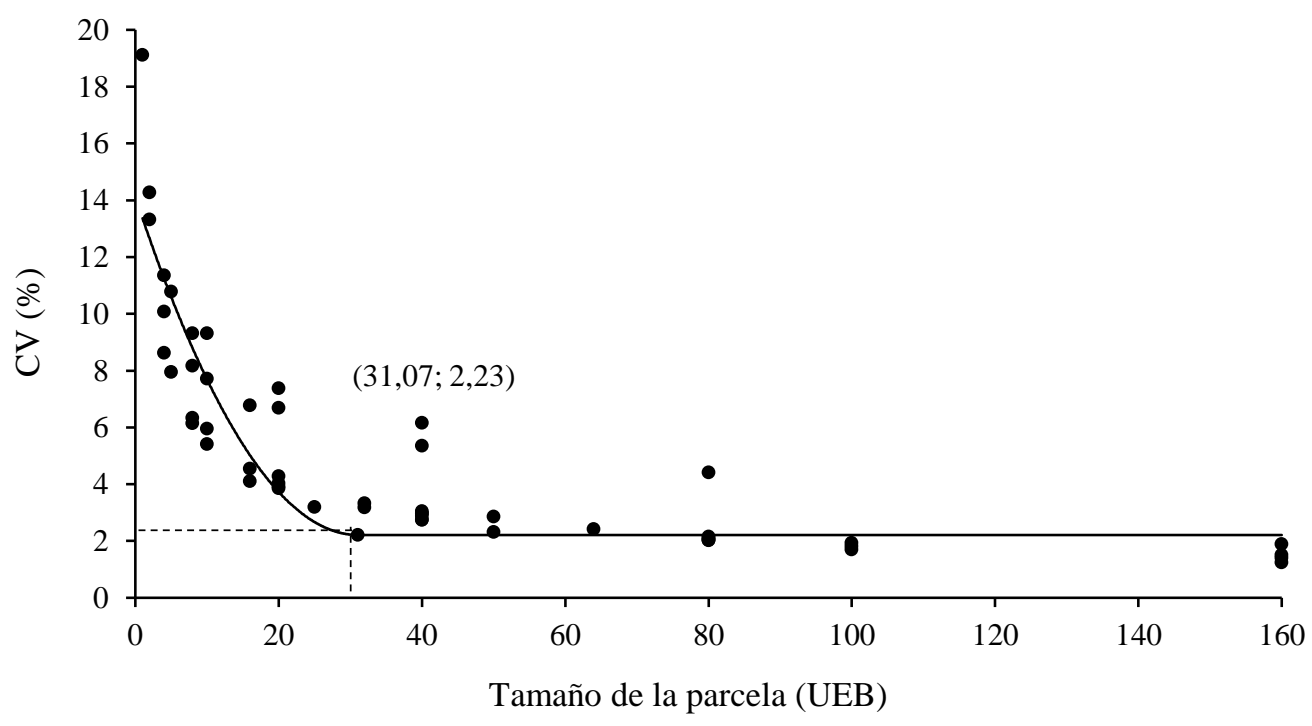

Figura 6. Ajuste mediante el modelo de regresión cuadrática con constante (QRP).

Este modelo tiene un coeficiente de determinación de $76.96 \%$ y un estadístico $\mathrm{F}$ también significativo ( $p<0.01$ ) (Cuadro 2). El tamaño de la parcela tiende a ser más alto con el método QRP que con el LRP, debido a la diferencia en el ajuste. Peixoto et al. (2011) en ensayos con maracuyá le atribuyen esta tenencia a la curvatura del modelo. Mendes et al. (2016) encontraron un tamaño de parcela para café de 10.53 UEB más grande con el método QRP respecto al método LRP. 
Por otro lado, Silva et al. (2012) utilizando un ensayo de rábano, encontraron una diferencia de 2.57 UEB a favor de método QRP. En ambos casos se aplicó el contraste de normalidad de ShapiroWilk (Shapiro y Wilk, 1965) a los errores del modelo $\left(\varepsilon_{\mathrm{i}}\right)$, tanto para el modelo LRP como para el QRP, y no se rechaza la hipótesis nula de normalidad con $(p=0.1998)$ y $(p=0.2181)$, respectivamente. Mediante los métodos de regresión segmentada se encontraron tamaños de parcela entre 72 y $93 \mathrm{~m}^{2}$.

Álvarez (1982) recomendó que el tamaño de las parcelas de investigación en caña de azúcar oscilara entre 80 y $115 \mathrm{~m}^{2}$ (5 o 6 surcos de ancho de 8 o $9.6 \mathrm{~m}$ ), con un largo de 10 a $12 \mathrm{~m} \mathrm{y}$ parcelas de 4 surcos de $12 \mathrm{~m}$ de largo $\left(76.8 \mathrm{~m}^{2}\right)$ en los casos en que existiera alguna limitación técnica o práctica (insumos, semilla, terreno, etc.). Ese autor aplicó el análisis de regresión múltiple y el método de curvatura máxima bivariada, sin encontrar grandes diferencias entre los dos métodos y en ambos casos, con coeficientes de determinación superiores $80 \%$.

Aunque los resultados aquí obtenidos se asemejan a los encontrados por Álvarez (1982), la comparación debe tomarse con reservas, debido a que se trata de una investigación que se llevó a cabo en Guatemala y como el autor lo indica en el título de su trabajo, su resultado está sujeto a las condiciones de la finca Bulbuxya, que se ubica en el departamento de Suchitepéquez de la región sur-occidente de la República de Guatemala y la inferencia, más allá de dicha zona, no es válida. Otro aspecto importante es la vigencia de este tipo de investigaciones.

Además, son necesarias las actualizaciones periódicas. Ese trabajo es de inicios de los años ochenta y no hay registros de actualización alguna. En Guatemala, Palencia (1965), empleó el método de Smith (1938) y calculó un tamaño óptimo de $27.24 \mathrm{~m}^{2}$, con lo que redondeó y recomendó una parcela útil de $28 \mathrm{~m}^{2}$ (dos surcos de $8 \mathrm{~m}$ de largo con separación de 1.8 a $2 \mathrm{~m}$ ), mucho más pequeñas que en el caso anterior. La parcela total sería de 4 surcos de $10 \mathrm{~m}$ de largo para excluir los dos surcos laterales y un metro en cada extremo de los surcos.

\section{Conclusiones}

El tamaño óptimo de la parcela experimental calculado con el método de regresión lineal con

constante fue de $72 \mathrm{~m}^{2}$ y con el método de regresión cuadrática con constante de $93 \mathrm{~m}^{2}$. Si se considera la variación que tienen los demás factores que no fueron incluidos en esta estimación, entonces, con el fin de minimizar el error experimental, se recomienda para ensayos de rendimiento que el tamaño de la parcela experimental con caña de azúcar debe estar entre 72 y $93 \mathrm{~m}^{2}$ y ajustar la forma de la parcela según la disponibilidad de espacio.

Se recomienda replicar la metodología aquí desarrollada en las demás regiones cañeras.

\section{Agradecimientos}

Al Departamento de Investigación y Extensión de la Caña de Azúcar (DIECA) de la Liga Agrícola Industrial de la Caña de Azúcar (LAICA) y a CoopeAgri, RL por los recursos y conocimiento aportados para el desarrollo de esta investigación. 


\section{Literatura citada}

Álvarez, V. M. 1982. Determinación del tamaño óptimo de parcela experimental en caña de azúcar bajo condiciones de la finca Bulbuxya. Tesis. Universidad de San Carlos de Guatemala, Guatemala. 59 p.

Bose, S. S. and Khanna, K. L. 1939. Note on the optimun shape and size of plots for sugarcane experiments in Bihar. The Indian J. Agric. Sci. 9(6):807-817.

Boyhan, G. E. 2013. Optimum plot size and number of replications for determining watermelon yield, fruit size, fruit firmness, and soluble solids. HortScience. 48(9):1200-1208. Doi: 10.21273/HORTSCI.48.9.1200.

Cocco, C.; Boligon, A. A.; Andriolo, J. L.; Oliveira, C. S. e Lorentz, L. H. 2009. Tamanho e forma de parcela em experimentos com morangueiro cultivado em solo ou em hidroponia. Pesquisa Agropecuária Brasileira. 44(7):681-686. Doi: 10.1590/S0100204X2009000700005.

Facco. G.; Cargnelutti, A. F.; Mendonça, B. A.; Lavezo, A.; Follmann, D. N.; Marques, C.; Schabarum, D. E.; Kleinpaul, J. A.; Görgen, G.; Lixinski, D.; Martins, F.; Barbieri, D. and Wartha, C. A. 2017. Basic experimental unit and plot sizes with the method of maximum curvature of the coefficient of variation in sunn hemp. Afr. J. Agric. Res. 12(6):415-423. Doi: 10.5897/AJAR2016.11814.

Ferreira, D. 2007. Uso de recursos computacionais. Lavras. Minas Gerais, Brasil. http://www.dex.ufla.br/ danielff/meusarquivospdf/RC0.pdf.

Giacomini, B. and Lúcio, A. 2018. Uniformity trials size for estimating cherry tomato plot size. Rev. Ciênc. Agron. 49(4):653-662. Doi:10.5935/1806-6690.20180074.

Igue, T.; Espironelo, A.; Cantarella, H. e Nelli, E. J. 1991. Tamanho e forma de parcela experimental para cana-de-açúcar. Bragantia. 50(1):163-180. Doi: 10.1590/S000687051991000100016.

Khan, M.; Hasija, R. C. and Tanwar, N. 2017. Optimum size and shape of plots based on data from a uniformity trial on Indian mustard in Haryana. Mausam. 68(1):67-74.

Krysczun, D.; Lúcio, A.; Sari, B.; Diel, M.; Olivoto, T.; Silva, J. Da; Santana, C.; Melo, P. and Gomes, S. 2018. The size of the uniformity trial affects the accuracy of plot size estimation in eggplant. J. Agric. Sci. 10(11):510-522. Doi: 10.5539/jas.v10n11p510.

Mamani. L. 1971. Determinación del tamaño forma y repetición de la parcela para ensayos de rendimiento en frijol (Phaseolus vulgaris L.). Tesis de maestría. Instituto Interamericano de Ciencias Agrícolas de la OEA. Turrialba, Costa Rica. 83 p.

Mendes, J.; Ferreira, A.; De Oliveira, J. M.; Silva, D.; Ribeiro, M. C. e Bortolini, J. 2016. Parcela ótima para a cultura do cafeeiro obtido por simulação de dados com variâncias conhecidas. PUBVET. 10(9):636-642. Doi: 10.22256/pubvet.v10n9.636-642.

Páez, G. 1962. Estudio sobre el tamaño y forma de parcela para ensayos en café. Tesis de maestría. Instituto Interamericano de Ciencias Agrícolas de la OEA. Turrialba, Costa Rica. 128 p.

Palencia, J. 1965. Determinación del tamaño óptimo de parcela experimental para estudios experimentales en caña de azúcar, bajo las condiciones de la estación experimental agrícola "Sabana Grande". Tesis de pregrado. Universidad de San Carlos de Guatemala, Guatemala. $42 \mathrm{p}$.

Paranaiba, P.; Ferreira, D. e Morais, A. 2009. Tamanho ótimo de parcelas experimentais: proposição de métodos para estimacão. Rev. Bras. Biometria. 27(2):255-268. 
Peixoto, A.; Faria, G. e Morais, A. 2011. Modelos de regressão com platô na estimativa do tamanho de parcelas em experimento de conservação in vitro de maracujazeiro. Ciência Rural. 41(11):1907-1913. Doi: 10.1590/S0103-84782011001100010.

Rodríguez, R.; Nogueira, C.; Rosales, R.; Silva, P. e Moraes, H. 2018. Tamaño óptimo de parcela $\mathrm{y}$ número de repeticiones para evaluar el rendimiento de boniato con mulch y suelo descubierto. Agrociencia Uruguay. 22(1):90-97. DOI: 10.31285/agro.22.1.9.

Santos, A. dos; Peixoto, C.; Almeida, A.; Santos, J. dos. e Machado, G. 2015. Tamanho ótimo de parcela para a cultura de girassol em três arranjos espaciais de plantas. Rev. Caatinga. 28(4):265-273. Doi: 10.1590/1983-21252015v28n430rc.

SAS. Institute. 2008. SAS/STAT User's guide. Version 9.3. Ed. Cary: SAS Institute.

Schwertner, D.; Lúcio, A. and Cargnelutti-Filho, A. 2015. Size of uniformity trials for estimating the optimum plot size for vegetables. Hortic. Bras. 33(3):388-393. Doi: 10.1590/S0102053620150000300019.

Shapiro, S. and Wilk, M. 1965. An analysis of variance test for normality (complete samples). Biometrika. 52(3/4):591-611. Doi: 10.2307/2333709.

Silva, L. F. da; Campos, K. A.; Morais, A. R. de; Cogo, F. D. e Zambon, C. R. 2012. Tamanho ótimo de parcela para experimentos com rabanetes. Rev. Ceres. 59(5):624-629. Doi: 10.1590/S0034-737X2012000500007.

Silva, W. C. da. 2014. Estimativas de tamanho ótimo de parcelas experimentais para a cultura do taro (Colocasia esculenta). Tesis Doctorado. Universidade Federal de Viçosa, Viçosa. $59 \mathrm{p}$.

Smith, H. F. 1938. An empirical law describing heterogeneity in the yields of agricultural crops. J. Agric. Sci. 28(1):1-23.

Sripathi, R.; Conaghan, P.; Grogan, D. and Casler, M. 2017. Field design factors affecting the precision of ryegrass forage yield estimation. Agron. J. 109(3):858-869. Doi: 10.2134/agronj2016.07.0397.

Vargas, J. y Navarro, J. 2014. Determinación de un tamaño adecuado de unidad experimental, utilizando el método de curvatura máxima, para ensayos de arroz (Oryza sativa), en Bagaces, Guanacaste. Revista Electrónica de las Sedes Regionales de la Universidad de Costa Rica. 15(31):128-144. Doi: 10.15517/ISUCR.V15I31.16018.

Vargas, J. y Navarro, J. 2017. Determinación del tamaño y la forma de unidad experimental, con el método de curvatura máxima, para ensayos de rendimiento de maíz (Zea mays). Guanacaste, Costa Rica. Cuadernos de Investigación. 9(1):135-144. 